\title{
High Energy Jets in the Earth's Magnetosheath: Implications for Plasma Dynamics and Anomalous Transport ${ }^{\mathrm{II}}$
}

\author{
S. Savin ${ }^{a}$, E. Amata ${ }^{b}$, L. Zelenyi ${ }^{a}$, V. Budaev ${ }^{c}$, G. Consolini ${ }^{b}$, R. Treumann ${ }^{d}$, E. Lucek , $^{e}$ \\ J. Safrankova $f$, Z. Nemecek, Y. Khotyaintsev ${ }^{g}$, M. Andre ${ }^{g}$, J. Buechner ${ }^{h}$, H. Alleyne, \\ P. Song $j$, J. Blecki ${ }^{k}$, J. L. Rauch ${ }^{l}$, S. Romanov ${ }^{a}$, S. Klimov ${ }^{a}$, and A. Skalsky ${ }^{a}$ \\ ${ }^{a}$ Space Research Institute, Russian Academy of Sciences, Moscow, 117997 Russia \\ e-mail:ssavin@iki.rssi.ru \\ ${ }^{b}$ Istituto Nazionale di Astrofisica-Istituto di Fisica dello Spazio Interplanetario, 00133 Roma, Italy \\ ${ }^{c}$ Nuclear Fusion Institute, Russian Research Centre Kurchatov Institute, Moscow, 123182 Russia \\ ${ }^{d}$ Munich University, Munich, 80539 Germany \\ ${ }^{e}$ The Blackett Laboratory, Imperial College London, London SW7 2AZ, UK \\ ${ }^{f}$ Charles University, 11636 Praha 1, Czech Republic \\ ${ }^{g}$ Swedish Institute of Space Physics, SE-751 21 Uppsala, Sweden \\ ${ }^{h}$ Max-Planck-Institut für Sonnensystemforschung, 37191 Katlenburg-Lindau, Germany \\ ${ }^{i}$ University of Sheffield, Sheffield S10 2TN, UK \\ ${ }^{j}$ University of Massachusetts, Lowell, MA 01854, USA \\ ${ }^{k}$ Space Research Center, Polish Academy of Sciences, 00716 Warsaw, Poland \\ ${ }^{l}$ Laboratoire de Physique et Chimie de l'Environnement, F-45071 Orleans, France
}

Received April 10, 2008; in final form, April 29, 2008

\begin{abstract}
High energy density jets in the magnetosheath near the Earth magnetopause were observed by Interball-1 [1]. In this paper, we continue the investigation of this important physical phenomenon. New data provided by Cluster show that the magnetosheath kinetic energy density during more than one hour exhibits an average level and a series of peaks far exceeding the kinetic energy density in the undisturbed solar wind. This is a surprising finding because the kinetic energy of the upstream solar wind in equilibrium should be significantly diminished downstream in the magnetosheath due to plasma braking and thermalization at the bow shock. We suggest resolving the energy conservation problem by the fact that the nonequilibrium jets appear to be locally superimposed on the background equilibrium magnetosheath, and, thus, the energy balance should be settled globally on the spatial scales of the entire dayside magnetosheath. We show that both the Cluster and Interball jets are accompanied by plasma superdiffusion and suggest that they are important for the energy dissipation and plasma transport. The character of the jet-related turbulence strongly differs from that of known standard cascade models. We infer that these jets may represent the phenomenon of the general physical occurrence observed in other natural systems, such as heliosphere, astrophysical, and fusion plasmas [2-10].
\end{abstract}

PACS numbers: 52.40.Hf, 52.30.-q, 52.40.-w

DOI: $10.1134 / \mathrm{S} 0021364008110015$

The region downstream of a supercritical collisionless shock, the magnetosheath (MSH), is known to be in a highly disturbed turbulent state [1-3]. The undisturbed solar wind (SW) streams with supermagnetosonic velocity $V>c_{\mathrm{ms}}$ at a magnetosonic Mach number up to $M_{\mathrm{ms}} \sim 15$. At the Earth's bow shock (BS), the SW decelerates to Mach numbers $M_{\mathrm{ms}}<1$, thermalizes, and, when entering the $\mathrm{MSH}$, is compressed by roughly a factor of 4. The flow downstream of the BS is highly disturbed and turbulent. However, the MSH is not spacious enough for the turbulence to reach a quasi-sta-

\footnotetext{
${ }^{\text {II }}$ The text was submitted by the authors in English.
}

tionarity. It remains not fully developed, intermittent, and structured in time and space. In this framework, high-energy density jets have been observed in the past in the magnetosheath $[1,5]$. As a development of such earlier studies, we have found more than 140 events of an anomalously high kinetic energy density in the MSH during 20 orbits of Interball-1, Cluster, Polar, and Geotail. Here, we concentrate on two MSH crossings-by Interball-1 and Cluster [11], respectively-characterized by the bursts of an extraordinarily high ion flux and kinetic energy density.

Figure 1 shows an example of the intermittent MSH jets [15] as observed by Interball-1 on March 29, 1996. 


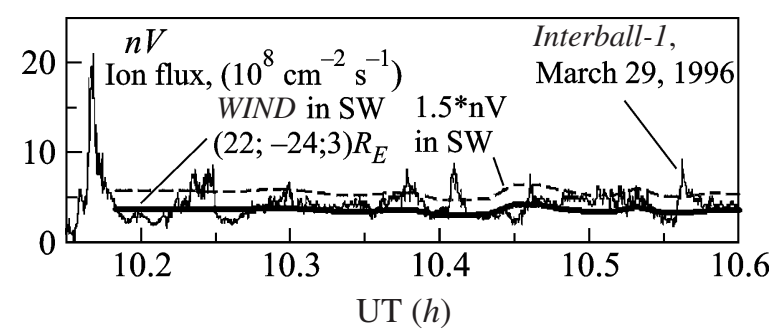

Fig. 1. (Thin line) ion flux in the turbulent boundary layer (TBL) near the magnetopause as measured by Interball-1 at (4.6; $2.8 ; 10.2) R_{E}$ GSM on March 29, 1996. The thick line shows the SW ion flux measured by WIND at $(22 ;-24 ; 3)$ $R_{E}$. The WIND flux multiplied by 1.5 is also shown by the dashed line as a rough MHD proxy for the MSH flow.

The thin line shows the plasma flux measured by the onboard Faraday cup instrument [5] with a time resolution of 1/16 s. The plasma flow provides direct estimates for the plasma transport (cf. Fig. 4). The thick line gives the SW upstream flow from WIND, while the dashed line represents a proxy for the MSH flow from the MHD prediction. One can see a number of ion flux spikes much higher than the equilibrium MSH flow (dashed line). Excluding the jets, the observed flux appears to be lower than expected in the MSH by a factor of $\sim 1.5$, since it roughly matches the SW flux. Similar matching to the SW flux has been found in the middle MSH for about 10 Interball-1 and Cluster cases. Thus, the jets look to carry the flux difference, providing the flow balance towards the MHD prediction.

Figure 2 shows kinetic energy density $W_{k}$, plasma density $N$, velocity $V$, and magnetic pressure $W_{b}$ from the Cluster 1 spacecraft and SW kinetic energy density $W_{\mathrm{kSW}}$ from WIND from 09:00 to 11:00 UT on March 27, 2002, when Cluster entered the magnetosheath inbound from the SW close to the southern magnetospheric cusp region. This MSH crossing has been analyzed previously in view of the reconnection in the MSH at scales of $\sim 100 \mathrm{~km}$ corresponding to thin current sheets [12], while, here, we concentrate mostly on scales larger by at least one order of magnitude. Cluster enters the MSH from the SW at about 09:35 UT. The SW as seen by the WIND spacecraft outside the foreshock at this time was very quiet with a kinetic energy density practically constant at $\sim 4.5 \mathrm{keV} / \mathrm{cm}^{3}$, as shown by the thick curve in the lower panel. The SW can be considered uniform over the distance between WIND and the Sun-Cluster line $\left(\sim 8 R_{E}\right)$. The average SW speed, particle, and kinetic energy densities were $\left\langle V_{\mathrm{sw}}\right\rangle \sim 470 \mathrm{~km} / \mathrm{s},\left\langle N_{\mathrm{sw}}\right\rangle \sim 4 \mathrm{~cm}^{-3}$, and $\left\langle W_{\mathrm{ksw}}\right\rangle \sim$ $4.5 \mathrm{keV} / \mathrm{cm}^{3}$. Compared to the SW (first $5 \mathrm{~min}$ on the left-hand side of Fig. 2), the MSH plasma exhibits an extremely high level of fluctuations. As shown in the central panels of Fig. 2, the $W_{k}$ peaks result from a combination of peaks in $N$ and in $V^{2}$ and are usually dominated by those in $N$, corresponding to plasma compres-
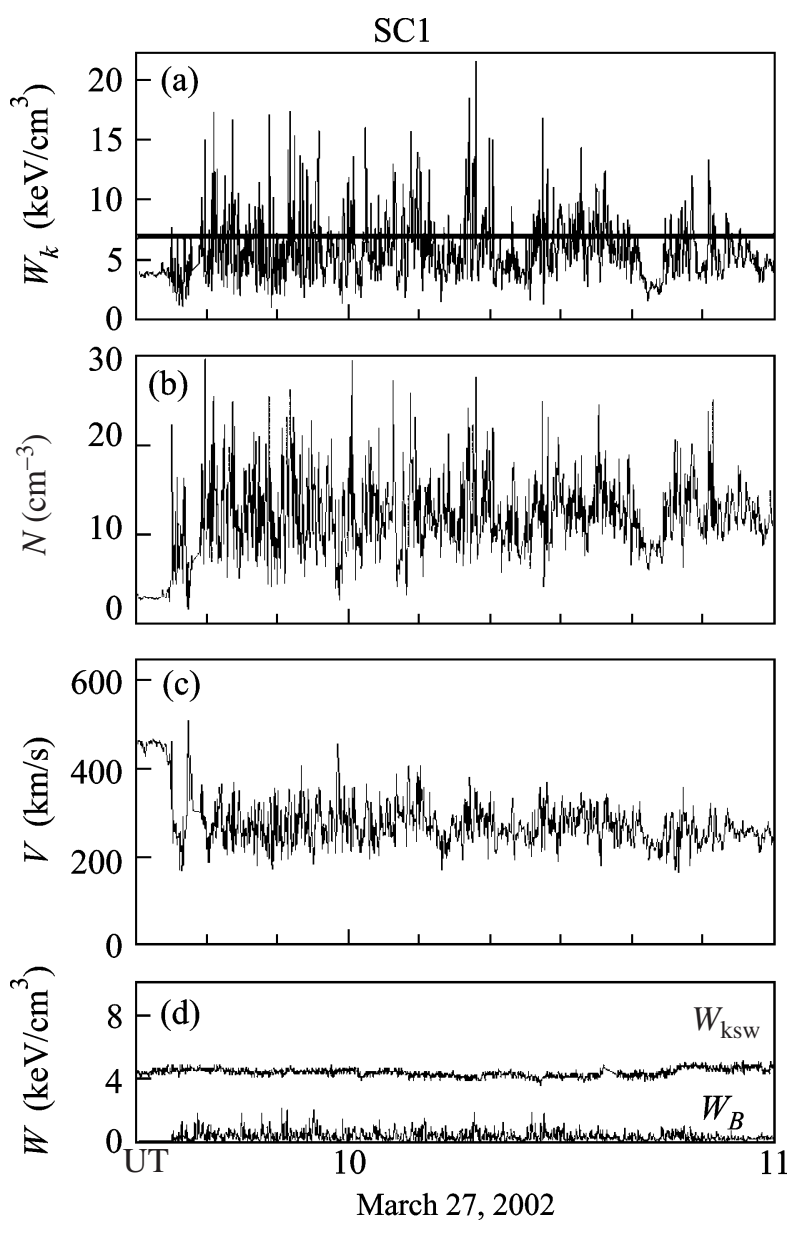

Fig. 2. Cluster $1 \mathrm{MSH}$ crossing of March 27, 2002 (see the orbit in [12]; GSM $Y \sim-6 R_{E}$ ). Panels (a)-(c) show a 4-s resolution kinetic energy density $W_{k}=0.5 m_{p} N V^{2}$ ( $m_{p}$ being the proton mass), ion density $N$, and velocity $V$. Panel (d) shows the kinetic energy density measured by WIND in the solar wind and the Cluster 1 magnetic pressure $W_{b}=B^{2} / 2 \mu_{0}$. The horizontal thick line in panel (a) corresponds to the vertical dashed line defined in Fig. 3.

sions moving at enhanced MSH speeds. After $11 \mathrm{UT}$, the level of MSH fluctuations greatly decreased, while the upstream solar wind plasma parameters did not change significantly.

Figure 3 shows the probability density functions (PDFs) of the MSH kinetic energy density for the 09:40-10:40 UT period, i.e., the central part of Fig. 2 characterized by the large $W_{k}$ peaks, and for 11:00 11:54 UT (the points on the dashed line), which we use as a reference period for a quieter MSH. It is clear that the two MSH regimes are completely different, while the solar wind energy density was the same in the two cases. A Gaussian equilibrium distribution is found to fit the "quiet" 11:00-11:54 UT PDF (dashed line). Conversely, for the 09:40-10:40 UT period, the PDF shows a clear non-Gaussian shape with a tail extending to very large values of the energy density. The strong asymme- 


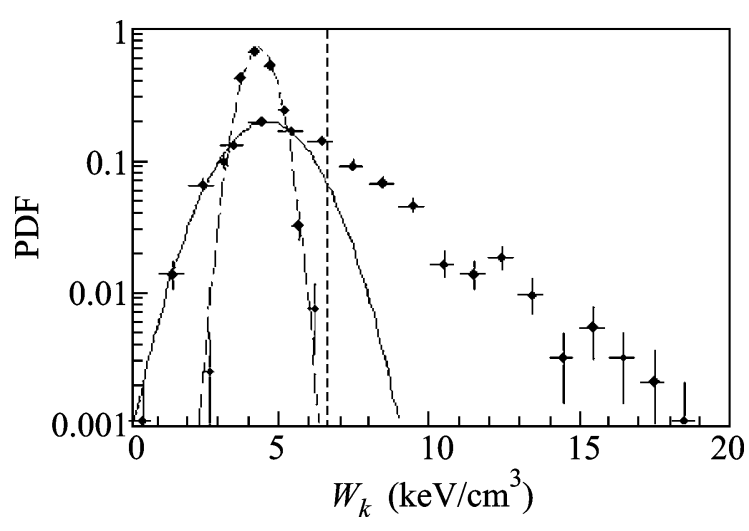

Fig. 3. Semi-log plot of the probability density functions (PDFs) of the MSH kinetic energy density $W_{k}$ for two periods: (points with error bars) 09:40-10:40 UT and (points on the dashed line) 11:00-11:54 UT. The dashed and solid line are Gaussian best fits of the 11:00-11:54 UT PDF and of the 09:40-10:40 UT PDF left side $\left(W_{k}<6 \mathrm{keV} / \mathrm{cm}^{3}\right)$, respectively. The vertical dashed line indicates the threshold chosen for the detection of jets.

try of the 09:40-10:40 UT PDF points towards the presence of an extra nonequilibrium contribution arising from the plasma jets: a Gaussian fit, similar to that of 11:00-11:54 UT PDF, can be performed only for the left-hand side $\left(W_{k}<6 \mathrm{keV} / \mathrm{cm}^{3}\right)$ of the 09:40-10:40 UT PDF (solid curve), whose peak $W_{k}^{0}$ and variance $\sigma_{G}$ are such that $W_{k}^{0}+1.5 \sigma_{G}=6.7 \mathrm{keV} / \mathrm{cm}^{3}$. This energy den- sity is marked as a vertical dashed line, which falls above all of the PDF points for the 11:00-11:54 UT period. Therefore, it looks reasonable to consider the $W_{k}=6.7 \mathrm{keV} / \mathrm{cm}^{3}$ threshold as the maximum kinetic energy density, which one should expect for the equilibrium MSH plasma between 09:40 and 10:40 UT and draw it as a horizontal line in Fig. 2a. We note that the $0<W_{k}<6.7 \mathrm{keV} / \mathrm{cm}^{3}$ interval contains more than $68 \%$ of the $W_{k}$ values and that the chosen threshold is $\sim 1.5 W_{\mathrm{kSW}}$ (where $W_{\mathrm{kSW}}$ is the $\mathrm{SW}$ kinetic energy density).

Turning back again to Fig. 2, we notice that the thick threshold line is exceeded by quite a number of peaks in $W_{k}$ by a factor of up to 3 . In the following, we concentrate our analysis on such peaks, which we call "high-kinetic energy density plasma Jets" (HKPJ). This definition is based on a much higher threshold in the kinetic energy density than for the earlier published cases of enhanced MSH flows [1,4].

Under the stable SW conditions monitored by WIND, this quantitative definition infers that all HKPJs be of an MSH or BS origin. The close inspection of the peaks has led to a count of 83 HPKJs during the period under study, having an average duration of 28 s (i.e., $\sim 6000 \mathrm{~km}$ ). In 77 cases, a velocity increase relative to the ambient MSH is also seen. In 26 cases, density enhancements are seen close to the HKPJ edges suggesting a piling up of the ambient MSH plasma. In 57 cases, the $N$ and $V$ peaks do not coincide. The $W_{k}$ peak corresponds in time to the $N(V)$ peak in 29 (10) cases.
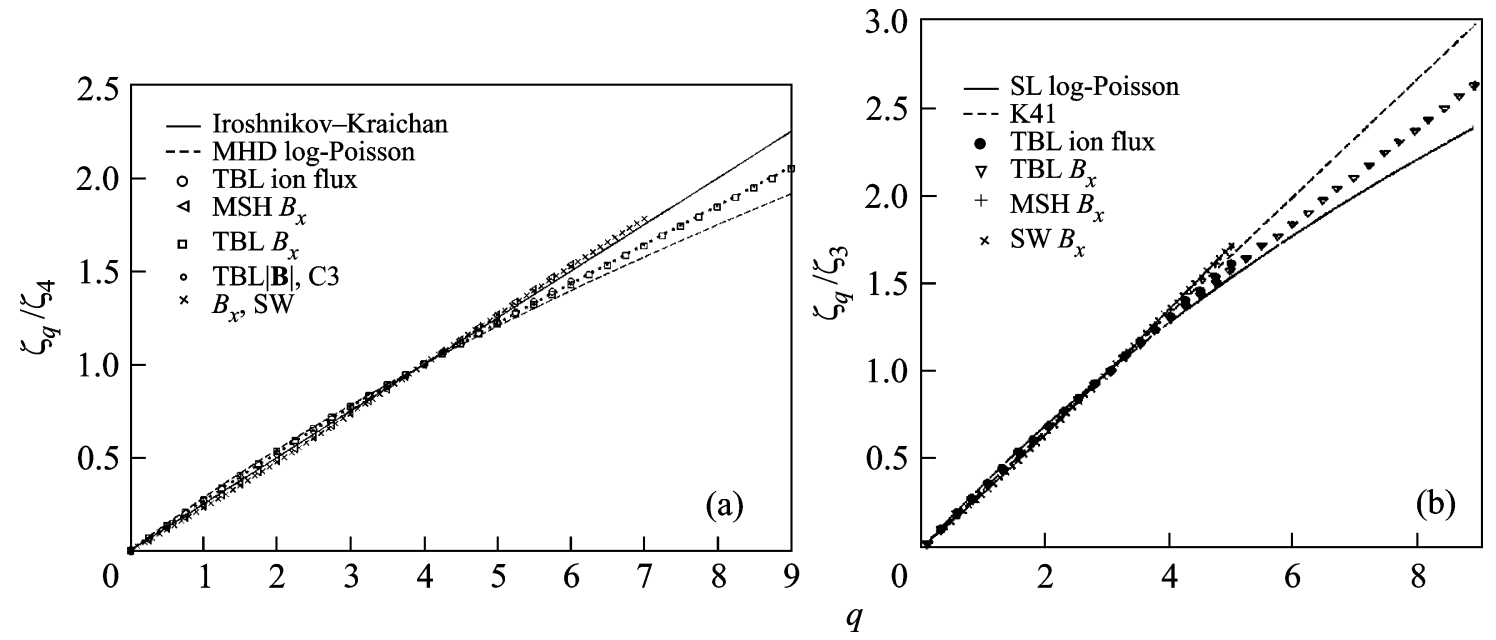

Fig. 4. (a) Relative exponent $\zeta(q) / \zeta(4)$ dependence on its order $q$ for the Interball-I (TBL ion flux, MSH $B_{x}$, TBL $B_{x}$ ), Cluster 3 (TBL $|\mathbf{B}|, \mathrm{C} 3)$, and Geotail $\left(B_{x}, \mathrm{SW}\right)$ data (see the respective symbols in the right-bottom corner and the text for details). The solid line is the Iroshnikov-Kraichnan scaling $q / 4[23,24]$. The dashed line is the log-Poisson model of the She-Leveque modified in the MHD case to account for the IK phenomenology [27]. (b) Dependence of the scaling ratio $\zeta(q) / \zeta(3)$ on its order $q$ versus that of Kolmogorov K41 (a dashed line) and that of the log-Poisson She-Leveque model (SL, [28]) describing the developed 3D turbulence (a solid line). The symbols in the right-bottom corner mark different experimental signals for the Interball-I (TBL ion flux, MSH $B_{x}$, TBL $\left.B_{x}\right)$ and Geotail $\left(B_{x}, \mathrm{SW}\right)$ data. 
We note that a part of the SW kinetic energy transforms at the BS into the thermal one, yielding a decrease of the kinetic energy density $W_{\mathrm{kSW}} \sim$ $4.5 \mathrm{keV} / \mathrm{cm}^{3}$, which one can compare with $\left\langle W_{k}\right\rangle \sim$ $6 \mathrm{keV} / \mathrm{cm}^{3}$ (with the standard variance $\sigma=2.6$ ) in the disturbed MSH. We address this paradox as follows: the equilibrium MSH subpopulation with $\left\langle W_{\mathrm{kMSH}}\right\rangle$ $4 \mathrm{keV} / \mathrm{cm}^{3}$ (see dashed line Fig. 3) is superimposed by the transient jets, having $W_{k}>6.7 \mathrm{keV} / \mathrm{cm}^{3}$ and respective measured $\left(W_{k J}\right) \sim 9 \mathrm{keV} / \mathrm{cm}^{3}(\sigma \sim 2.4)$. Thus, one gets approximately the measured average value $\left\langle W_{k}\right\rangle$ for the mixture of equilibrium and nonequilibrium subpopulations. Really, this is not a simple mixture of noninteracting plasmas, since, in the disturbed region, the parallel and perpendicular ion temperatures are equal and have a much wider spread into the lower magnitude region, while, in the quiet $\mathrm{MSH}$, the perpendicular temperature strongly dominates (not shown). The jets most probably are transient both in space and time (cf. Figs. 1-3); thus, the energy conservation should be settled only on a time interval over several characteristic time intervals for the jets and only at a spatial scales comparable with the entire dayside MSH. The latter conforms to the fact that the ion flux near the magnetopause (see Fig. 1) has the background level much below the MHD proxy, contrary to the ion flux at 09:40-10:40 UT (cf. Fig. 2) being on average about 1.5 times higher than its MHD proxy from the SW WIND data (not shown). That is, the flux closer to MP (recall that Fig. 1 also includes about 10 such cases mentioned above) can be considerably smaller than the MHD proxy, while the flux just inside BS could conversely exceed the MHD proxy. For a further quantitative check of this point, one needs to find a fortunate case in the Cluster and THEMIS data and run MHD model.

Considerations, similar to that of $W_{k}$, are valid also for the respective Mach numbers $M_{\mathrm{ms}}$ : between 11:00 and 11:20 UT, $\left\langle M_{\mathrm{ms}}\right\rangle \sim 1(\sigma \sim 0.5)$; between 09:42 and 11:00 UT, $\left\langle M_{\mathrm{ms}}\right\rangle \sim 1.34(\sigma \sim 0.27)$. The latter suggests that a supermagnetosonic population with $W_{k}>$ $6.7 \mathrm{keV} / \mathrm{cm}^{3}$ and $\left\langle M_{\mathrm{ms}}\right\rangle \sim 1.62$ with $\sigma \sim 0.25$ adds to that with low $M_{\mathrm{ms}}$ giving the above-mentioned average value.

Further to the analysis we carried out for $W_{k}>$ $6.7 \mathrm{keV} / \mathrm{cm}^{3}$, we concentrated on 33 stronger HKPJs having $W_{k}>10 \mathrm{keV} / \mathrm{cm}^{3}$ (i.e., $>1.5 \sigma$ over the $\left\langle W_{k}\right\rangle$ in the $\mathrm{MSH})$, for which we studied the distribution of angles $\alpha=a \sin \left(V_{z} /|\mathbf{V}|\right)$ and found that for $36 \%$ of the cases $\mathbf{V}$ was deflected from the average MSH flow towards the MP by $>16^{\circ}$, while for $12 \%$ of the cases $\mathbf{V}$ was deflected by $>34^{\circ}$. The strongest HKPJs have $\left\langle W_{k}\right\rangle \sim$ $16 \mathrm{keV} / \mathrm{cm}^{3}$ and a characteristic scale $\sim 6$ s, i.e., 1000 $1500 \mathrm{~km}$, which correspond to 3-11 proton gyroradii. Namely, these strongest HKPJs represent the most distinguishable new entities to which this Letter is devoted. It is most interesting to check the magnetic signatures of the HKPJs (displayed by the lower solid line in Fig. 2d). The magnetic pressure $W_{b}$ is very low during this passage of the MSH and is completely negligible compared with the kinetic energy density, in particular, when compared with $W_{k}$ in the jets. Moreover, in most of the observed jets, the magnetic field increases do not coincide with the jet maxima, nor does the magnetic pressure exhibit a minimum in the jet centers (which would be required for a plane current layer). This makes it difficult to associate the jets uniquely with thin fast-current sheets [13]. The important conclusion from this comparison is that a reconnection cannot be the cause of the HKPJs simply because there is no sufficient energy stored in the magnetic field.

The observation of such high energy density jets in the intermittent turbulence of the MSH plasma [13] poses a serious problem to understanding the possible mechanisms of their formation. In this regard, we note that the dynamic interaction in the MSH plasma, which is bounded at one side by the BS and at the opposite side by the MP, is nonuniform and intrinsically transient, since the plasma is still evolving from the shocked to a statistically equilibrium turbulent state. During the course of this evolution, it seems that processes may occur which concentrate the free energy in the still underdeveloped turbulence and focus the plasma into jets of a very high kinetic energy density in a way that is relatively independent of the state of the upstream SW. It is also probable that such processes are favored by the presence of moving boundaries [4]. How this proceeds, with an ion kinetic energy increase inside the jets being comparable with an ion temperature drop (cf. [18]), remains unclear. To that extent, we first recall a mechanism based on an inertial-drift plasma acceleration by nonuniform electric field structures $[1,4,19]$ almost standing in the MP frame, which can account for the enhanced-jet velocities. Such structures constitute wave interference patterns in the MSH [19] with an electric field forcing the incident flow into an equilibrium state that adjusts for the presence of the moving boundaries [4] and transfers the momentum downstream the MSH by means of the jet formation.

Now, we would like to discuss in further detail the implications of the HKPJs for the turbulence and transport characteristics. As mentioned above, the substantial part of the HKPJs in Fig. 2 can hit the MP, and all such HKPJs with $W_{k}>6.7 \mathrm{keV} / \mathrm{cm}^{3}$ pierce through the downstream MP (cf. [30]), having a total pressure (magnetic + thermal) well below $6 \mathrm{keV} / \mathrm{cm}^{3}$. In addition, we suggest that such jets, detected in front of an MP (see Fig. 1 and [1]), with their specific statistical properties could provide the respective diffusion-like transport across the MP. Thus, further on we make use of the statistical properties of different signals in the extended regions with HKPJs (cf. Figs. 1,2) to explore the transport properties (self-similarity scalings) in this important region. 
Table

\begin{tabular}{l|c|c|c}
\hline \multicolumn{1}{c|}{ Data type } & $\Delta$ & $\beta$ & $K(-1)$ \\
\hline TBL near MP, $B_{x}$, Interball-1 & 0.24 & 0.38 & 0.39 \\
TBL near MP, ion flux, Interball-1 & 0.2 & 0.36 & 0.36 \\
TBL downstream BS, $|\mathbf{B}|$, Cluster 3 & 0.23 & 0.41 & 0.33 \\
MSH, $B_{x}$, Interball-1 & $\approx 0$ & 1 & $\approx 0$ \\
SW, $B_{x}$, Geotail (simultaneously with TBL near MP, $\left.B_{x}\right)$ & $\approx 0$ & 1 & $\approx 0$ \\
\hline
\end{tabular}

The statistical properties are studied by analyzing the structure functions (i.e., the moments of the probability distribution function (PDF); see Fig. 3 and, e.g., [6]) of different orders of $q$ versus time lag $\tau$ from the experimental time series $X(t): S_{q}(\tau)=\left\langle\left|\delta_{\tau} X(t)\right|^{q}\right\rangle$, $\delta_{\tau} X(t)=X(t+\tau)-X(t)$, where $\langle\ldots\rangle$ stands for the statistical averages, from the experimental time series $X(t)$. A statistical self-similarity of the type $S_{q}(\tau) \sim \tau^{\zeta(q)}$ can be expected; $m$ is the inertial range. For the isotropic fully developed 3D turbulence (described by Kolmogorov's K41 model [22]), the scaling exponents $\zeta(q)=q / 3[3$, 15]. We analyze the data, displayed in Figs. 1 and 2 (at 09:40-10:40 UT on March 27, 2002), by fitting parameters $\beta$ and $\Delta$ of a log-Poisson turbulent cascade model [25] for different experimental scalings:

$$
\zeta(q)=(1-\Delta) \frac{q}{3}+\frac{\Delta}{1-\beta}\left[1-(\beta)^{\frac{q}{3}}\right],
$$

where the $\beta$ and $\Delta$ parameters characterize intermittency and singular dissipative structures, respectively. For 3D isotropic turbulence, Z.S. She and E. Leveque (SL) have proposed $\beta=\Delta=2 / 3$ [5]. The IroshnikovKraichnan model $[23,24]$ leads to a reduction of the problem symmetry. A Kolmogorov-type energy spectrum in magnetized plasma can also be derived in an assumption regarding the critical balance [26]. The logPoisson model was modified for the MHD case to account for the IK phenomenology [27] (MHD IK). This phenomenology depends on the dimension of the most intensely dissipative structures and their scaling (the IK model supposes two-dimensional sheet-like dissipative structures [27]).

To test the IK hypothesis, in Fig. 4a, we display the relative exponent $\zeta(q) / \zeta(4)$ dependence from the $q$ for different spacecraft data (cf. [15]). The scaling of a GSE magnetic component $B_{x}$ in MSH outside TBL on June 19,1998 , is close to the IK scaling $q / 4$. The same is true for the SW $B_{x}$ from Geotail recorded at the same time interval on June 19,1998 , as the $B_{x}$ in TBL on Interball- $1[1,15]$. It infers that, generally, the BS does not substantially change the SW statistical properties. In contrast to the simultaneous SW data, the TBL $B_{x}$ data from Interball-1 [1] are deviated strongly from the IK scaling. For the extremely disturbed TBL on Cluster 3 closer to the BS (cf. Fig. 2), surprisingly one sees a practically coincident scaling with that of Interball-1 near the MP.

In the finite pressure plasma, the magnetic fluctuations could have different properties from that of the ion flow. But, the scaling of the ion flow in TBL (see the TBL ion flux in Fig. 4a and cf. Fig. 1) well fits that of the $B_{x}$. Figure 4 a demonstrates that the original IK phenomenology does not describe the scaling property of the intermittent turbulence in the TBL. At the same time, they are not fitted by the MHD IK scaling [27]. Thus, the TBL turbulence is neither isotropic nor does it have 2D dissipative structures.

We also look for a power-law dependence of $S_{q}(\tau)$ on $S_{3}(\tau), S_{q}(\tau) \sim S_{3}(\tau)^{\zeta(q) / \zeta(3)}$, i.e., for an extended selfsimilarity (ESS, [15]). In the cases under study, [15] demonstrates the ESS properties. We compare the scaling of the $S_{q}$ with the K41 and the SL models. The quiet MSH and SW scalings deviate slightly more from the K41 straight line than that of Fig. 4a from the IK straight line. The TBL scalings differ from that of the K41 turbulence as much as from the IK scaling. $\zeta(q) / \zeta(3)$ in the TBL also departs from the SL model [28]. Instead, [15] demonstrates that the TBL dissipative structures are most likely the 1D filaments.

We have checked the plasma transport properties in the jet regions by analyzing the particular cases displayed in Figs. 1, 2, 4 and published in [1]. For that purpose, we display the fitted the log-Poisson parameters $\beta$ and $\Delta$ in the table. The data in [14] approximates the scaling of the diffusion coefficient as $\mathscr{D}_{f} \propto \tau^{K(-1)}$, where $K(q)=q-\zeta(3 q)$. It is a result of considering the average over various initial walker starting positions; this is equivalent to an ensemble averaging.

From the table for scaling (1), one gets $K(-1) \approx$ $0.33-0.39$. The average displacement of a particle scales as: $\left\langle\delta x^{2}\right\rangle \propto \mathscr{D}_{f} \tau \propto \tau^{\Psi}$, with $\Psi=1+K(-1) \approx 1.33-$ $1.39>1$, which infers the superdiffusion. Note that, for the classical diffusion, $\Psi=1$, as is the case for the MSH and SW $B_{x}$ (table, two bottom rows).

On the other hand, we also fitted the probability distribution of $\delta_{\tau}|\mathbf{B}|$ and $\delta_{\tau} V_{z}$ from Cluster by a Levy function $L_{\alpha}=(1 / \pi) \int \exp \left(-\gamma \tau^{\alpha}\right) \cos (\tau x) d x$ at various $\tau$.

In particular, corresponding to the proton cyclotron frequency, we choose [17] $\tau=1.4 \mathrm{~s}$ and the fits yield the parameter [16-18] $\alpha \sim 1.66$ or $\sim 1.8$, which give [17] 
$\langle\delta x\rangle^{2} \propto t^{2 / \alpha}=t^{1.2}$ or $t^{1.11}$ for the Cluster $\delta_{\tau}|\mathbf{B}|$ and $\delta_{\tau} V_{z}$, respectively. For the Interball case, the Levy function fitting for the magnetic vector rotation angles gives [16] $\alpha \sim 1.2$, and, respectively, $\langle\delta x\rangle^{2} \propto t^{1.67}$.

Finally, a further analysis was performed between 09:40 and 10:40 UT based on HIA Cluster 3 ion energy [11], $\epsilon=0.5 m_{p}(\delta \mathbf{V})^{2}$, where $\delta \mathbf{V}=\mathbf{V}-\langle\mathbf{V}\rangle$ and $\mathbf{V}$ is the 4 s HIA ion velocity (cf. Fig. 2). By applying the rankordering statistics [21] to the $\epsilon$ time series, we get [17] $\langle\delta x\rangle^{2} \propto t^{1.11}$.

We would like to outline that, in our scaling analysis, we have excluded small scales comparable or less than on the order of proton inertial length [13] (by the respective averaging or using data with a low sampling rate), which gives unrealistic $\left(\langle\delta x\rangle^{2}\right)$ time scalings with a power exponent over $2[17,14]$.

In spite of the scaling exponent spread, all of these scalings correspond to the superdiffusion [17]. Thus, qualitatively, we conclude that the MSH turbulence including HKPJs exhibits signs of the plasma superdiffusion and anomalous plasma transport. It should affect the effective deceleration of the MSH flow closer to the MP (i.e., dissipate the kinetic energy in boundary layers) via carrying the downflow of the momentum "excess" by the jets $[1,19]$, supporting the SW plasma penetration across the high-latitude MP. Note that about $20 \%$ of the strongest jets on March 27, 2002 (see Fig. 2 and discussion above) are considerably deflected towards the MP from the average direction of the MSH flow, and, hence, these jets with $W_{k} \gg W_{b}$ at the MP could provide the superdiffusive transport inside the high-latitude magnetosphere.

One could consider the possibility that the turbulent reconnection, as proposed for the March 27, 2001 event $[12,1]$, provides a method for the free flow of the supermagnetosonic jets (with an interior Mach number $\left.\left\langle M_{\mathrm{ms}}\right\rangle \sim 1.6\right)$ across the subsonic background $\mathrm{MSH}$, although the ambient magnetic-field energy density cannot provide a substantial amount of energy density for the jet generation. Kuznetsov et al. [29] proposed the Alfvénic collapse under high ion- $\beta$ conditions (which holds in Fig. 2) to provide the local plasma acceleration through the expulsion of plasma by the collapsing magnetic fields. As for Fig. 2, the Alfvénic collapse is not seen in the lower panel (thus, a local acceleration via the Alfvénic collapse can be excluded), so that the secondary reconnection at the jet borders looks to be operative. Similar phenomena are known from random "hot spots" in the laser plasmas [20], which initiate the two-dimensional self-focusing of laser beams into filaments.

To conclude this Letter, we recall that interacting moving matters in the laboratory, in fusion plasmas as in the tokamak $[9,15]$ boundary layer or laser plasmas [20], in the heliosphere and also in astrophysical plasmas $[6,7]$ frequently generate localized jets with a high kinetic energy density [3-10] exceeding the kinetic-, thermal-, and magnetic-field energy densities of the interacting components. Such systems are quite different from each other, as regards their plasma parameters and the physical processes which occur in them; nevertheless, they seem to display the collimating of the kinetic energy into narrow spatial regions as a common property. The dynamic plasma interaction advanced in this paper differs from the classic one [31]. The analysis of Interball and Cluster data indicates a more sophisticated scenario of the plasma flow braking. Before the final (asymptotic) plasma thermalization downstream from the BS, a number of localized HKPJ structures are formed within the MSH, which could have numerous important dynamical implications discussed above.

The correspondence between the jets and Alfvénic/magnetosonic [13] eigenstates, which are localized in the density humps, is analogous to the correspondence between the discrete eigenstates of the one-dimensional nonlinear Schrödinger equation whose stationary solutions are solitons [20].

In most cases, the jet-flux amplitude and jet duration indeed resemble a standard flow quantization. Savin et al. [1] suggested that the maser-like flow quantization by self-focused jets is due to the transition of a flow from a metastable state with a super-Alfvénic velocity to a stable state with Alfvénic or sub-Alfvénic flows. The latter state permits the magnetic-stress balance (i.e., a force-free equilibrium) that minimizes the total energy of the flow-obstacle interaction. As a consequence, the MSH flux aims at the SW flux levels (cf. the background $W_{k}$ in Fig. 1).

This was also the case on June 19, $1998[1,19]$. Thus, the jets can provide a considerable input into a quasi-static flow balance in the MSH. This is a task for future investigation to illuminate the interrelation between the above mechanisms of the jet generation for the different types of jets $[4-7,15]$.

Nature seems to "prefer" highly nonuniform equilibriums with large excesses of free (in our case, kinetic) energy. The mechanism of jet generation is still barely understood; its further study should shed light on the inherent transient dynamics of plasma streaming [6, $7,15]$ and its transport properties.

This work was partially supported by INTAS (project nos. 03-50-4872, 06-100017-8943, and 051000008-8050), the Russian Foundation for Basic Research (project nos. 06-02-17256, 07-02-92210NTNIL, and 07-02-00319), International Space Science Institute (Bern, Switzerland), and the State Committee for Scientific Research in Poland (grant nos. 8T12 E 01628 and NN 307065834). We are grateful to Prof. G.N. Zastenker for providing the Interball1 ion flux data and to the MFI team for providing the Geotail high-resolution magnetic data. We thank A. Volosevich, S. Zhestkov, E. Churazov, and S. Moiseenko for helpful discussions. 


\section{REFERENCES}

1. S. P. Savin, L. M. Zelenyi, E. Amata, et al., JETP Lett. 79, 452 (2004).

2. D. G. Sibeck et al., Space Sci. Rev. 88, 207 (1999).

3. F. Sahraoui, G. Belmont, L. Rezeau, et al., Phys. Rev. Lett. 96, 075002 (2006).

4. E. Amata, S. Savin, M. Andre, et al., Nonlin. Proc. Geophys. 13, 365 (2006).

5. Z. Nemecek, J. Safrankova, L. Prech, et al., Geophys. Res. Lett. 25, 1273 (1998).

6. M. C. Begelman, R. D. Blandford, and M. J. Rees, Rev. Mod. Phys. 56, 255 (1984).

7. A. I. MacFadyen, S. E. Woosley, and A. Heger, Astrophys. J. 550, 410 (2001).

8. D. Bartolo, C. Josserand, and D. Bonn, Phys. Rev. Lett. 96, 124501 (2006).

9. V. P. Budaev, S. Takamura, N. Ohno, and S. Masuzaki, Nucl. Fusion 46, S181 (2006).

10. J. Merka, J. Safrankova, Z. Nemecek, et al., Adv. Space Res. 25, 1425 (2000).

11. The Cluster Mission Description, Ann. Geophys. 19, 1197 (2001).

12. A. Retino, D. Sundkvist, A. Vaivads, et al., Nature Physics 3, 236 (2007).

13. D. Sundkvist, A. Retino, A. Vaivads, and S. D. Bale, Phys. Rev. Lett. 99, 025004, (2007).

14. A. V. Chechkin, R. Gorenflo, and I. M. Sokolov, Phys. Rev. E 66, 046129 (2002).

15. V. P. Budaev, S. Savin, L. Zelenyi, et al., Plasma Phys. Contr. Fusion. 50 (2008), DOI /10.1088/07413335/50/1.
16. S. Savin, J. Buechner, G. Consolini, et al., Nonlin. Proc. Geophys. 9, 443 (2002).

17. R. A. Treumann, Geophys. Res. Lett. 24, 1727 (1997).

18. S. Savin, L. Zelenyi, E. Amata, et al., A Planet. Space Sci. 53, 133 (2005).

19. S. Savin, A. Skalsky, L. Zelenyi, et al., Surv. Geophys. 26, 95 (2005).

20. P. A. Robinson, Rev. Mod. Phys. 69, 508 (1997).

21. D. Sornette, Critical Phenomena in Natural Sciences. Chaos, Fractals, Selforganization and Disorder: Concepts and Tools (Springer, Berlin, Heidelberg, 2000).

22. A. N. Kolmogorov, Dokl. Akad. Nauk SSSR 30 (4), 299 (1941); Proc. R. Soc. London, Ser. A 434, 9 (1991).

23. P. S. Iroshnikov, Astron. Zh. 40, 742 (1963) [Sov. Astron. 7, 566 (1964)].

24. R. H. Kraichnan, Phys. Fluids 8, 1385 (1965).

25. B. Debrulle, Phys. Rev. Lett. 73, 959 (1994).

26. P. Goldreich and S. Sridhar, Astrophys. J. 438, 763 (1995).

27. W. Muller and D. Biskamp, Phys. Rev. E 67, 066302 (2003).

28. Z. S. She and E. Leveque, Phys. Rev. Lett. 72, 336 (1994).

29. E. A. Kuznetsov, S. Savin, F. Amata, et al., JETP Lett. 85, 288 (2007).

30. J. Lemaire and M. Roth, Space Sci. Rev. 57, 59 (1991).

31. R. Z. Sagdeev, Reviews of Plasma Physics, Vol. 4, Authorized translation from the Russian by Herbert Lashinsky, University of Maryland, USA, Ed. by M. A. Leontovich (Consultants Bureau, New York, 1966), p. 23. 